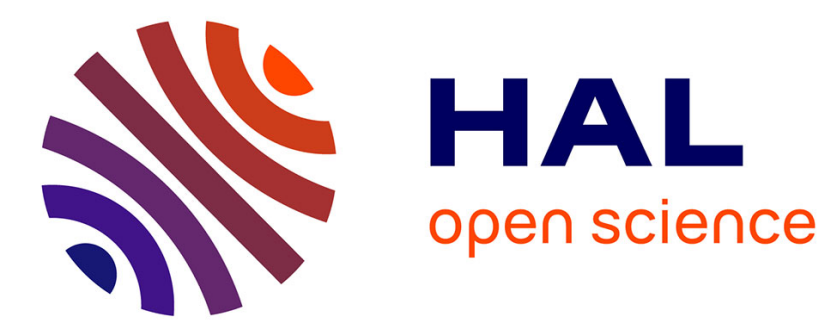

\title{
Production de champs magnétiques transitoires de l'ordre de 130 teslas (1,3 mégaoersted) par décharge de batterie de condensateurs
}

\author{
M. Guillot
}

\section{- To cite this version:}

M. Guillot. Production de champs magnétiques transitoires de l'ordre de 130 teslas (1,3 mégaoersted) par décharge de batterie de condensateurs. Revue de Physique Appliquée, 1976, 11 (4), pp.541-545. 10.1051/rphysap:01976001104054100 . jpa-00244089

\section{HAL Id: jpa-00244089 https://hal.science/jpa-00244089}

Submitted on 1 Jan 1976

HAL is a multi-disciplinary open access archive for the deposit and dissemination of scientific research documents, whether they are published or not. The documents may come from teaching and research institutions in France or abroad, or from public or private research centers.
L'archive ouverte pluridisciplinaire HAL, est destinée au dépôt et à la diffusion de documents scientifiques de niveau recherche, publiés ou non, émanant des établissements d'enseignement et de recherche français ou étrangers, des laboratoires publics ou privés. 


\title{
PRODUCTION DE CHAMPS MAGNÉTIQUES TRANSITOIRES DE L'ORDRE DE 130 TESLAS (1,3 MÉGAOERSTED) PAR DÉCHARGE DE BATTERIE DE CONDENSATEURS
}

\author{
M. GUILLOT $\left({ }^{*}\right)$ \\ Laboratoire de magnétisme, C. N. R. S., BP 166, Centre de Tri, 38042 Grenoble, Cedex, France
}

(Reçu le 12 février 1976, accepté le 4 mars 1976)

\begin{abstract}
Résumé. - Description de la technique de production de champs magnétiques intenses transitoires atteignant 1,3 MOe (130 T) par décharge de batterie de condensateurs (énergie voisine de $35 \mathrm{~kJ}$ ) dans une bobine monospire de $5 \mathrm{~mm}$ de diamètre utile. Le comportement de la bobine sous l'effet des efforts électrodynamiques et de l'échauffement est étudié ; l'intérêt de bobines construites en tantale est démontré.
\end{abstract}

Abstract. - An apparatus producing high transient magnetic fields up to $1.3 \mathrm{MOe}(130 \mathrm{~T})$ is described; the capacitor bank energy is of the order of $35 \mathrm{~kJ}$; the inside diameter of the single turn coil is $5 \mathrm{~mm}$. The stability of the coil under mechanical and thermal action of the field is studied : tantalum appears to be the best material for resisting repeated generation of very high fields.

\section{Notations et unités.}

$H(t)$ : valeur instantanée du champ en Oe $\left(^{1}\right)$. $I(t)=\frac{V}{L^{\prime} W}(\exp -\mu t) \sin w t:$ courant de décharge en $A$.

$\mu=\frac{R^{\prime}}{2 L^{\prime}}$ constante d'amortissement en $\mathrm{s}^{-1}$.

$w=\frac{2 \pi}{T}=\left(\frac{1}{L^{\prime} C}-\mu^{2}\right)^{1 / 2}:$ pulsation en $\mathrm{s}^{-1}$.

$a_{1}$ rayon intérieur en $\mathrm{cm}$.

$a_{2}$ rayon extérieur en $\mathrm{cm}$.

$2 b$ longueur en $\mathrm{cm}$.

$m$ : masse spécifique g. $\mathrm{cm}^{-3}$.

$T_{\mathrm{f}}$ température de fusion en degrés Kelvin.

$\rho$ : résistivité en $\Omega . \mathrm{cm}$.

$C_{\mathrm{v}}$ : chaleur spécifique en $\mathrm{J} . \mathrm{cm}^{-3}$.

$s:\left(\frac{10^{9} \rho}{4 \pi w}\right)^{1 / 2}:$ couche de peau en $\mathrm{cm}$.

$\alpha: \frac{a_{1}+S}{a_{1}}$.

$\beta: \frac{2 b}{a_{1}}$.

$\left(^{*}\right)$ Expériences réalisées en collaboration avec le Centre d'Etudes Nucléaires de Grenoble (Laboratoire des Applications Spéciales de la Physique) et le Service National des Champs Intenses de Grenoble.

(1) L'unité de champ magnétique utilisée est l'oersted (l'induction correspondante s'exprime en $\mathrm{G}$ par le même nombre dans un milieu non magnétique $\left.\left(\mu_{0}=1\right)-1 \mathrm{~T}=10^{4} \mathrm{G}\right)$.
1. Introduction. - Les techniques de production de champs magnétiques égaux ou supérieurs au mégaoersted, basées sur la compression de flux sont autodestructives : inconvénient quasi inévitable, puisque produit par la focalisation d'un tube métallique lancé à des vitesses de l'ordre du $\mathrm{km} \mathrm{s}^{-1}$ [1]. Si cette situation est jugée inacceptable, il faut abandonner la compression de flux et revenir au principe même des champs pulsés (décharge d'une batterie de condensateur dans un solénoïde), considérant que les limites habituelles de cette technique, situées vers $600 \mathrm{kOe}$, sont susceptibles d'être considérablement améliorées [2].

$100 \mathrm{~T}(1 \mathrm{MOe})$ correspondent à une densité d'énergie magnétique de $4 \mathrm{~kJ} \mathrm{~cm}^{-3}$, valeur qui représente environ $50 \%$ de la densité d'énergie d'un explosif chimique [3]. Le champ se comporte dans la bobine comme un gaz à deux dimensions et exerce sur la surface intérieure une pression radiale $H^{2} / 8 \pi$ (40 kbar pour $1 \mathrm{MOe}$ ) supérieure aux limites élastiques de tous les conducteurs.

Sous de telles pressions, on peut admettre qu'une fois dépassée la limite élastique, la matière se comporte comme un fluide et que seule la force d'inertie s'oppose à l'expansion du solenoïde. Le temps $t$ nécessaire au champ pour propulser le métal sur sa propre épaisseur $a_{2}-a_{1}$ est de l'ordre de :

$$
\bar{t}=\frac{a_{2}-a_{1}}{\bar{v}}=\frac{4\left(a_{2}-a_{1}\right)}{H}(\pi m)^{1 / 2} .
$$

Pour $m=10 \mathrm{~g} \mathrm{~cm}^{-3}$ et $H=2 \mathrm{MOe}$, la vitesse 
TABleau I $(*)$

Fonctions des paramètres géométriques permettant le calcul des champs maximum pour deux types de densité de courant

$\begin{array}{cc}\begin{array}{c}\text { Densité } \\ \text { de courant } \\ \text { dans couche } \\ \text { de peau }\end{array} & \frac{H(\mathrm{Oe})}{I(\mathrm{~A})} \\ \text { Uniforme } & \frac{1}{a_{1}} \cdot 0,7897 \cdot \frac{1}{2 \beta(\alpha-1)} \cdot F(\alpha, \beta) \\ 1 / r & \frac{1}{a_{1}} \cdot 0,7897\left(\frac{1}{\beta \ln \alpha}\right)^{1 / 2} G(\alpha, \beta)\end{array}$

* Voir référence [12].

moyenne $\bar{v}$ est de l'ordre du $\mathrm{mm} \mathrm{s}^{-1}$ et $\bar{t}$ est égal à $10 \mu \mathrm{s}$ pour $a_{2}-a_{1}$ égal au centimètre. On pourrait déduire que pour contenir des champs de plus en plus élevés, il suffit d'accroître dans la même proportion $a_{2}-a_{1}$. L'expérience désavoue totalement cette conclusion : une partie de la couche conductrice tend à rester en arrière de la zone accélérée, d'où l'apparition d'arêtes et de plis qui au cours des prochaines décharges seront des points privilégiés pour le développement d'amorçage. Ces instabilités sont alignées parallèlement au champ et ont une longueur d'onde de l'ordre du millimètre pour des champs de l'ordre du mégaœrsted, ce qui laisse à penser que le temps de formation est de quelques microsecondes [4, 5].

Il devient alors impératif de disposer d'impulsion de courant dans le domaine de la microseconde. Ceci impose un réservoir d'énergie (défini comme l'ensemble batterie de condensateurs, circuit de décharge, et connections diverses) de faible inductance et de faible capacité. En conséquence, le courant va circuler essentiellement sur la surface des conducteurs, la couche de peau (entre 0,1 et $2 \mathrm{~mm}$ ) étant le siège des efforts électrodynamiques et des échauffements ; une première estimation de l'élévation de température donnée par $3000 \mathrm{H}^{2}\left(\mathrm{MOe}^{2}\right)$ [6] montre que le point de fusion de la plupart des métaux sera atteint ou dépassé.

La bobine doit être à la fois de réalisation simple (elle se trouve à la limite de la destruction) et présenter une faible inductance ; ces conditions justifient l'orientation vers des enroulements monospire dont nous fixons le diamètre intérieur à $5 \mathrm{~mm}$, dimension nécessaire à la réalisation de mesures physiques.

Le calcul a priori de l'onde de courant est extrêmement complexe puisque les impédances (l'appareillage se résume à un circuit série $R^{\prime} L^{\prime} C$ ) sont fonctions de la couche de peau fonction elle-même de la fréquence et de l'échauffement (fréquence et échauffement étant déterminés par les impédances). A plus forte raison le calcul de l'onde de champ est encore plus délicat, puisque le rapport champ/courant dépend aussi de la couche de peau (Tableau I). Compte tenu de cette situation, nous considérons l'onde de courant comme une donnée obtenue à partir de l'expérience.

$$
\begin{array}{cc}
\text { Facteur géométrique } & R_{b} \\
- & - \\
F(\alpha, \beta)=\frac{2 \pi \beta}{5} \ln \frac{\alpha+\left(\alpha^{2}+\beta^{2}\right)^{1 / 2}}{1+\left(1+\beta^{2}\right)^{1 / 2}} & \frac{\rho \pi(\alpha+1)}{a_{1}(\alpha-1)^{2} \beta} \\
G(\alpha, \beta)=\frac{1}{5}\left(\frac{\pi}{\beta \ln \alpha}\right)^{1 / 2} \ln \alpha \frac{\beta+\left(1+\beta^{2}\right)^{1 / 2}}{\beta+\left(\alpha^{2}+\beta^{2}\right)^{1 / 2}} & \frac{\rho}{a_{1}} \cdot \frac{\pi}{\beta \ln \alpha}
\end{array}
$$

2. Description de l'appareillage. -2.1 LA BATTERIE DE CONDENSATEURS. - Le stockage de l'énergie s'effectue dans une batterie de condensateurs : capacité totale : $182 \mu \mathrm{F}$; la tension maximale de charge $20 \mathrm{kV}$ correspond à $36,4 \mathrm{~kJ}$. La commutation sur la bobine est assurée par un éclateur à air fonctionnant sous pression réduite $\left(5 \times 10^{-2}\right.$ à $10^{-2}$ torr $)$ de très faible inductance (entre 5 et $8 \mathrm{nH}$ ). Cet éclateur est constitué de plusieurs électrodes planes en tungstène séparées par des disques de polyéthylène. Une électrode des extrémités comporte l'igniteur auquel est appliquée l'impulsion de tension de $20 \mathrm{kV}$ (délivrée par un condensateur auxiliaire) qui provoque l'amorçage ; l'éclateur fonctionne pour des tensions de charge comprises entre 8 et $20 \mathrm{kV}$. Les connexions entre les éléments sont effectuées par 50 câbles coaxiaux de faible longueur ; l'une d'elles porte le transformateur toroïdal utilisé pour la mesure du courant de décharge.

2.2 L'ONDE DE DÉCHARGE. - Pour la bobine définie figure $1 a$, le régime de décharge est oscillatoire amorti avec $T / 4=3,8 \mu$ s et $\mu=0,835 \times 10^{5} \mathrm{~s}^{-1}$, soit $L^{\prime}$ et $R^{\prime}$ respectivement égaux à $30 \mathrm{nH}$ et $5 \times 10^{-3} \Omega$. Par différence avec les impédances de l'éclateur et des connexions, on déduit que l'inductance et la résistance de la bobine sont de l'ordre de $15 \mathrm{nH}$ et $2,5 \times 10^{-3} \Omega$.

Ces résultats sont en bon accord avec les valeurs calculées pour une densité de courant variant en $1 / r$ en prenant la résistivité du tantale constante au cours de la montée en champ et égale à sa valeur à $2000^{\circ} \mathrm{C}$ (qui correspond à la température moyenne du volume de peau pour un champ proche du mégaœrsted. Voir $\S 3.1)$. Notons que pour une densité de courant supposée uniforme, l'inductance calculée est beaucoup plus faible $(5 \mathrm{nH})$, alors que la résistance reste pratiquement constante.

2.3 RAPPORT ChAMP COURANT. - Considérant le courant nul à l'extérieur de la couche de peau, le champ correspondant est donné par les expressions du tableau I où $G(\alpha, \beta)$ et $F(\alpha, \beta)$ sont fonctions de la répartition du courant que nous supposerons soit uniforme, soit variant en $1 / r$. Pour le tantale, dans nos conditions expérimentales $S$ varie de $0,44 \mathrm{~mm}$ à la 


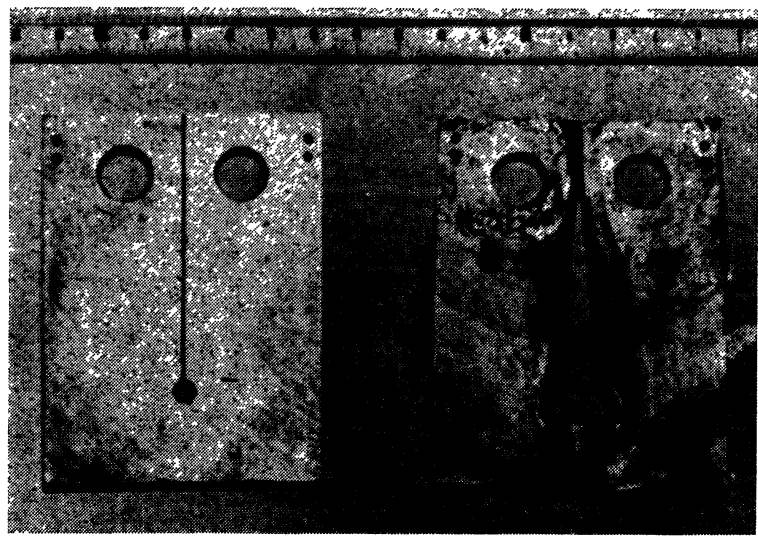

(a)

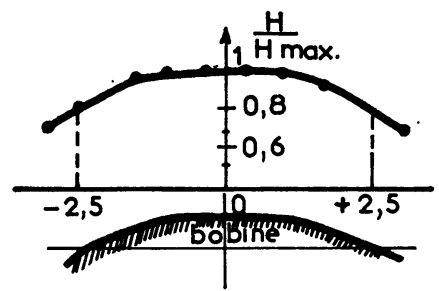

(c)

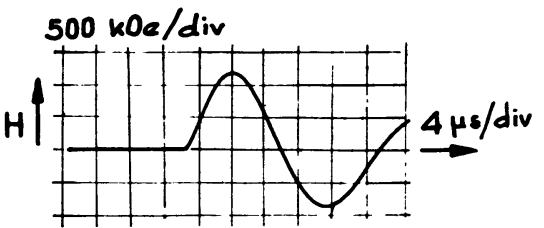

(d)

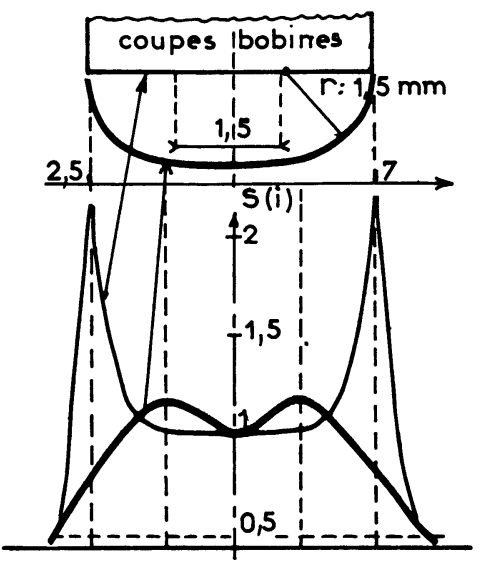

(e)

FIG. 1. - $-a)$-b) Bobine avant et après expérience. c) Uniformité axiale de champ. $d$ ). Onde de décharge $H(t)$. e) Courbes calculées des densités de courant.

\section{TABLEAU II}

Principales caractéristiques des matériaux susceptibles d'être utilisés pour la production de champs magnétiques intenses transitoires

$\begin{array}{ccccccc} & \begin{array}{c}m \\ \mathrm{~g} \mathrm{~cm}^{-3}\end{array} & T_{\mathrm{f}} & \begin{array}{c}\rho \\ \mu \mathrm{cm}\end{array} & \begin{array}{c}C_{\mathrm{v}} \\ \mathrm{J} \mathrm{cm}^{-3}\end{array} & \begin{array}{c}\text { Vitesse } \\ \text { du son } \\ 10^{5} \mathrm{~cm} \mathrm{~s}^{-1}\end{array} & \begin{array}{c}\text { Vitesse crit. } \\ 10^{3} \mathrm{~cm} \mathrm{~s}^{-1} \\ {[9]}\end{array} \\ \text { Tantale } & - & - & - & - & - & - \\ \text { Tungstène } & 16,6 & 3300 & 12,45\left(^{a}\right) & 2,30\left(^{b}\right) & 4,5 & 6,2 \\ \text { Acier } & 19,3 & 3700 & 5,3 & 2,6 & 5,2 & 0,4 \\ \text { Cuivre } & 7,8 & 1800 & 16 & 3,5 & 5,5 & 8 \\ & 8,9 & 1350 & 1,66 & 3,5 & 4,5 & 4,6\end{array}$

(a) $44,1 \mu \Omega$ à $1000^{\circ} ; 78,9$ à $2000^{\circ} ; 94,4$ à $2500^{\circ} ; 1087$ à $3000^{\circ} ; 115,5$ à $3269^{\circ}$ (réf. [13]).

(b) La variation thermique $C_{\mathrm{v}}=69,388\left(0,03322+0,4198 \times 10^{-5} \theta+0,3295 \times 10^{-1} \theta^{2}\right)$ (Réf. [12]).

température ambiante à $1,34 \mathrm{~mm}$ au voisinage du point de fusion (variation thermique de $\rho$ donnée Tableau II) ; on calcule que $H / I$ reste de l'ordre de 1,9 quelle que soit la distribution du courant : pour $a_{1}$ égal à $2,5 \mathrm{~mm}$, le mégaœrsted nécessite un courant de $550 \mathrm{kA}$, résultat confirmé avec une précision de $10 \%$ par l'expérience (cet écart provient très probablement des variations d'inductance et de résistance en cours de décharge aussi bien pour l'éclateur que pour la bobine).

3. Etude de la "monospire». - 3.1 Limitations DU CHAMP PAR L'ÉCHAUFFEMENT. - Compte tenu des intensités mises en jeu et de l'effet de peau, la fusion du volume de peau se produit par une valeur $H_{\mathrm{f}}$ du champ déduit de :

$$
\int_{0}^{t} R_{\mathrm{b}} I^{2} \mathrm{~d} t=V_{\text {peau }} C_{\mathrm{v}} T_{\mathrm{f}}=a_{1}^{3} \cdot 2 \pi \beta\left(\alpha^{2}-1\right) C_{\mathrm{v}} T_{\mathrm{f}} .
$$

En supposant $R_{\mathrm{b}}$ indépendant du temps (les variations thermiques de $\rho$ et $\alpha$ sont négligées) et une densité de courant en $1 / r$, l'intégration sur le temps de montée conduit à :

$$
\begin{aligned}
H_{\text {fusion }}=\sqrt{10^{9} C_{\mathrm{v}} T_{\mathrm{f}}} \times \exp ( & \left.-\mu \frac{\pi}{2 w}\right) \sqrt{\frac{\pi}{w} \cdot K} \times \\
& \times 2,51 \mathrm{G} \sqrt{\frac{\alpha+1}{2 \beta(\alpha-1)}}
\end{aligned}
$$


avec

$$
K(w, \mu)=\frac{\mu^{2}+w^{2}}{w^{2}\left[1-\exp \left(-\mu \frac{\pi}{w}\right)\right]-2 \mu^{2} \exp \left(-\mu \frac{\pi}{w}\right)}
$$

expression qui permet de tirer les conclusions suivantes. Le matériau de la monospire doit présenter un produit $C_{\mathrm{v}} T_{\mathrm{f}}$ le plus élevé possible, d'où l'orientation vers des métaux réfractaires (molybdène, tungstène, rhénium, tantale). La figure $2 a$ qui représente la variation du second terme en fonction de $\mu / w$ montre l'intérêt d'une onde de décharge avec $\mu / w$ compris entre 0,08 et $0,4(\mathrm{si} \mu / w$ est inférieur à 0,08 il est avantageux de mettre une résistance en série avec la bobine). Le dernier terme dépend avant tout de $\alpha$ et par suite de la résistivité et $d u$ temps de montée; pour une bobine quadratique $(\beta=1)$, il ne varie que de 0,59 à 0,55 lorsque $\alpha$ passe de 1,05 à 1,6 (Fig. $2 b$ ), on peut donc conclure que l'accroissement de la résistivité sous l'action de la température ne produit qu'une très faible diminution du champ.

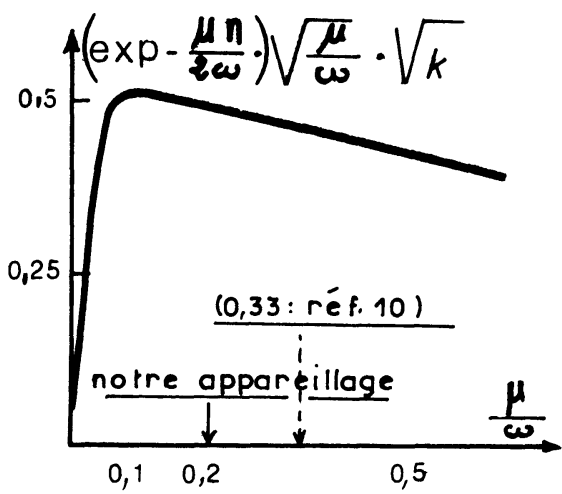

(a)

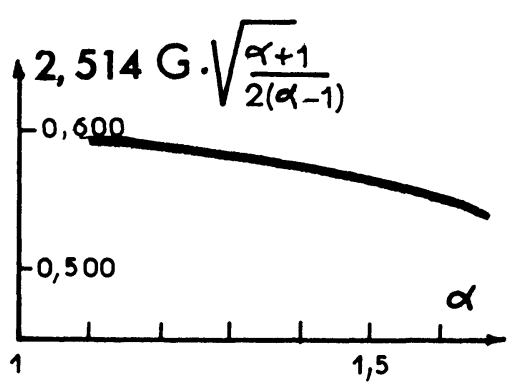

(b)

FIG. 2. $-a$ )-b) Courbes calculées des fonctions déterminant la valeur du champ à l'instant où se produit la fusion de la surface intérieure de la bobine.

Si l'échauffement est calculé sur la demi-période, le terme en $\mu^{2}$ disparaît du dénominateur de $K(w, \mu)$; et le champ est réduit d'environ $20 \%$.

Pour le tantale on obtient $\boldsymbol{H}_{\mathrm{f}}=950 \mathrm{kOe}$; nos conditions expérimentales $(\mu / w \simeq 0,2)$ correspondent à $900 \mathrm{kOe}$. Ces résultats sont très proches de ceux déduits des équations de Maxwell, $H_{\mathrm{f}}=\left(8 C_{\mathrm{v}} T_{\mathrm{f}}\right)^{1 / 2}$, qui montrent que tenant compte de l'effet transitoire, le premier demi-cycle d'une onde sinusoïdale donne seulement $70 \%$ de l'échauffement précédemment calculé [6]. On conclut que le tantale supporte les élévations de température liées à la présence des champs légèrement supérieurs au mégaœrsted et ce quelles que soient les dimensions de la bobine (une densité uniforme de courant conduit à la même conclusion). Ces valeurs sont à comparer à $H_{\mathrm{f}}$ de l'ordre de $600 \mathrm{kOe}$ pour l'alliage de cuivre à $2 \%$ de béryllium, habituellement utilisé en champ pulsé, et de 700 kOe pour un bon acier.

3. 2 OPTIMISATION DE LA FORME DE LA SPIRE. Lorsque la jonction du trou cylindrique et des deux faces parallèles de la plaque se fait à angle droit, une détérioration très rapide des extrémités du trou est constatée. L'étude expérimentale de l'uniformité du champ montre dans cette zone un accroissement du champ d'environ $15 \%$ par rapport à sa valeur au centre lié à la présence d'une densité de courant plus élevée. Le calcul effectué pour un courant permanent suffisamment faible pour éviter l'échauffement confirme cette hypothèse : la densité de courant $\delta(i)$ est le double de sa valeur au centre [7] (Fig. 1e). Par contre, pour une spire dont les angles sont arrondis, la densité de courant est beaucoup plus homogène et la température de surface de la spire devient à peu près uniforme (Fig. 1e), ce qui réduit les risques de destruction.

3.3 LES EFFORTS ÉLECTRODYNAMIQUES. - En plus de l'échauffement, le facteur qui détermine la durée de vie de la bobine est évidemment l'action des efforts électrodynamiques. L'effet de ces contraintes dépend avant tout pour un métal donné, de sa pureté, des traitements thermiques préalables à l'utilisation et des conditions d'usinage (nos monospires sont réalisées par les techniques d'électroérosion) et par suite, la comparaison de différents matériaux ne peut être que très qualitative.

Dans une première approximation, le métal peut être considéré comme imperméable au champ. Pour une impulsion sinusoïdale le déplacement de la couche de peau après une demi-période est donné par :

$$
x(\mathrm{~mm})=0,5 \frac{10^{-2} H_{0}^{2} T^{2}}{m S}
$$

à champ égal une impulsion courte apparaît comme favorable, ainsi que l'emploi de matériaux de masse spécifique élevée (on retrouve ainsi l'intérêt des métaux réfractaires).

Pour le tantale, $x$ est de l'ordre de $1,2 \mathrm{~mm}$ pour une période de $20 \mu \mathrm{s}$ (soit un accroissement de $25 \%$ du diamètre intérieur).

En réalité, le champ magnétique diffuse dans le conducteur. Un ordre de grandeur de la vitesse de diffusion de l'énergie magnétique peut être calculé en supposant au temps zéro le champ nul dans tout le conducteur (considéré comme un milieu infini à une 
dimension) sauf dans une mince couche où sa valeur est infiniment grande. L'énergie magnétique est concentrée dans une zone d'abscisse

$$
X(\mathrm{~mm})=\frac{3}{4} \times 10^{5}(2 \rho t)^{1 / 2}
$$

qui se déplace à une vitesse $X(2 t)^{-1}$. Pour les métaux bons conducteurs, cette vitesse est après $100 \mathrm{~ns}$, de l'ordre de $2 \mathrm{~mm} \mathrm{\mu s}^{-1}\left(2 \times 10^{5} \mathrm{~cm} \mathrm{~s}^{-1}\right)$, soit 30 à $50 \%$ de la vitesse du son [8].

Il apparaît alors que le choix du matériau doit s'effectuer à partir des propriétés mécaniques non pas du régime statique, mais du régime dynamique : résistance à l'impact, vitesse critique d'impact $v_{\mathrm{cr}}$ (définie en considérant que la destruction se produit aux points où la vitesse d'impact est supérieur à $v_{\mathrm{cr}}$ ) tout en recherchant de fortes masses spécifiques, puisque seule la force d'inertie s'oppose aux forces magnétiques [9]. L'acier, le cuivre et ses alliages au beryllium, le niobium et le tantale constituent les matériaux à $v_{\text {cr }}$ élevé $(1$ à $2 \%$ de la vitesse du son); parmi eux le tantale avec une masse spécifique très forte (double de celle de l'acier), constitue le meilleur choix (Tableau II). Notons que les autres métaux réfractaires qui du point de vue thermique apparaissaient comme très intéressants, sont à éliminer, compte tenu de leurs propriétés mécaniques (la vitesse critique du tungstène est environ $6 \%$ celle du tantale).

4. Champs produits : comportement de la bobine. $\mathrm{Au}$ cours des trois premières décharges, le champ magnétique atteint $1,3 \mathrm{MOe}$ avec un temps de montée de $3,8 \mu \mathrm{s}$. Le diamètre intérieur s'est alors accru d'environ $1 \mathrm{~mm}$. Après dix expériences à un mégaœrsted, le diamètre a augmenté d'environ $10 \%$ (ce qui allonge d'autant le temps de montée) et le champ diminue de $7 \%$; des trous de fusion apparaissent très nettement sur le diamètre intérieur, ainsi qu'une déformation de la fente. Ces effets s'aggravent très rapidement aux cours des expériences suivantes et la rupture totale de la spire se produit rapidement ( 3 à 5 tirs) Dans tous les cas, l'énergie utilisée est inférieure à $32 \mathrm{~kJ}$; la sonde de mesure et l'échantillon mesuré restent absolument intacts [3].

Sur un millimètre de part et d'autre du centre de la spire, l'uniformité axiale du champ est meilleure que l'incertitude sur la mesure du champ égale à $\pm 2 \%$; il en est de même pour l'uniformité radiale lorsqu'on s'écarte de l'axe sur 1,5 mm (Fig. 1c).

5. Conclusion. - Nous avons montré que des champs magnétiques non destructeurs du système de mesure et supérieurs au mégaœrsted pouvaient être obtenus par des batteries de condensateurs d'énergie raisonnables dans des monospires en tantale et ce de manière répétitive. L'accroissement des performances en champ vers 2,5 ou $3 \mathrm{MOe}$, peut être envisagé dans la mesure où les impulsions de courant seraient beaucoup plus brèves (temps de montée d'environ $2 \mu \mathrm{s}$ ) et le diamètre de la cavité de champ divisé par deux. Néanmoins, il est important de réaliser que dans ce cas, l'expérience devient quasi monocoup : le diamètre intérieur passant de 2 à $10 \mathrm{~mm}$ puisque la diffusion du conducteur est inévitable au-dessus du mégaœrsted [10, 11].

Remerciements. - Nous remercions MM. Marret et Sauneuf, ingénieurs au Service de Physique Générale du Centre d'Etudes de Limeil, pour l'aide efficace apportée à l'étude et à la réalisation de notre appareillage, ainsi que la Direction des Recherches et Moyens d'Essais, qui a financé en partie nos travaux.

\section{Bibliographie}

[1] Herlach, F., Reports on Progress in Physics, 1968, 31, p. 341.

[2] Les champs magnétiques intenses. Conférence de Grenoble 1966 (Edition du CNRS) no 166.

[3] GuIllot, M., Physique sous champs intenses. Conférence de Grenoble 1974 (Edition du CNRS) no 242.

[4] Kruskal, M., Schwarzchild, M., Proc. R. Soc. (London) A 223 (1954) 348.

[5] Golgate, S., 1955, U. C. R. L, 4560 Livermore.

[6] Furth, H. P., Levine, M. A., Waniek, R. W., Rev. Sci. Instrum. 28.11 (1957) 949.
[7] Marret, J. P., Sauneuf, R., Centre d'Etudes de Limeil. Communication Personnelle.

[8] Stratton, J. A. Théorie de l'électromagnétisme (Editions Dunod) 1961.

[9] Rinehart-Pearson, J. S., Behaviour of metals under impulsive loads, American Society of Metals, Cleveland 1954.

[10] Andrianov, A. M., Sh. E. T. F. ; Pis. Red. 11 p. (1970) 582.

[11] Forster, D. W., Martin, J. C., Réf. 2, p. 361.

[12] BRuCe Montgomery, D., Reports on progress in physics, 1963, Vol. XXVI, p. 69.

[13] Handbook of metals. American Phy. Soc. 1973. $8^{\mathrm{e}}$ Edition. 